\title{
Effects of raising rats on a liquid or solid diet on response choice made with hunger and thirst simultaneously operative'
}

ROBERT J. THOMPSON, JR., and THOMAS B. LEONARD, III, ${ }^{2}$ University of North Dakota, Grand Forks, N. D. 58201

One group of rats was raised normally on a diet of dry pellets, and another group was raised almost completely on a liquid diet of Metrecal, in order to control the amounts of reinforced time spent eating and drinking in each group, hence producing different incentive values for eating and drinking. Both groups were then trained to turn left in a T-maze for dry food and right for water. After $60 \mathrm{~h}$ of simultaneous hunger and thirst deprivation, free choice test trials were given. Normally raised rats always turned toward food, but rats raised on Metrecal turned toward food and water about equally often. The results are explained by the different incentive values for eating and drinking produced by the different diets.

It has been demonstrated that rats can learn to make different responses to two different drives (Heron, 1949; Hull, 1933; Leeper, 1935; Manning, 1954). Rats can learn to turn one way in a T-maze to get water when thirsty and the other way to get food when hungry. An interesting question arises: What response would an organism make if two drives were operating simultaneously, but the organism could choose to satisfy only one of the two drives?

The Hull-Spence theory predicts that the response to be made in such a choice situation will be that response which has the higher excitatory potential (Spence, 1956). However, two studies have apparently failed to support the prediction (Heron, 1949; Manning, 1954). On test trials for which the thirst drive equalled or exceeded the hunger drive and with approximately equal habit strengths for both drives, rats made approximately 100 per cent food responses. Assuming all other intervening variables in the Hull-Spence theory to have equal values for the two possible responses (food and water) it is clear that the theory predicts more water responses than food responses. The discrepancy between predicted and observed results suggests that other intervening variables may not have had equal values for the two responses.

This study examined the possibility that there was a difference in incentive values in favor of an eating response which develops in the normally raised rat. Since rats normally spend a longer time eating than drinking, a longer period of reinforcement is associated with eating than drinking, producing a higher incentive value for eating than drinking responses. To attempt to manipulate the incentive values of eating and drinking in rats two groups of rats were used. Following a suggestion of Kleinmuntz (1963) one group of rats was raised on a liquid diet (L), consisting of Metrecal and water, without any experience with dry food. Females with litters to be used in Group L were removed from the home cage for feeding. This group should have developed a stronger incentive value for drinking than eating, since eating, i.e., chewing and swallowing dry food, could not occur. A second group of rats was raised on a solid diet (S) consisting of dry food pellets and water. These rats should, according to the Hull-Spence theory, develop a greater incentive value for eating than drinking. Both groups were trained to turn left in a T-maze to receive food when hungry and right for water when thirsty. Then the rats were deprived of food and water simultaneously for $60 \mathrm{~h}$, at which time the two drives were approximately equal in strength (Crawford \& James, 1964), and a test of choice of response was made.

It was predicted that Group $S$ would make significantly more food choices than would be expected by chance, replicating previous findings, that Group $\mathrm{L}$ would make significantly more water responses than expected by chance, and that the choices of the two groups would differ significantly.

\section{SUBJECTS}

All rats were of the Sprague-Dawley strain. At the beginning of maze training the 21 rats of Group L, raised from the time of weaning on a Metrecal and water diet, were 38 days old, and the 20 rats of Group $S$, raised on a diet of dry food and water, were 40 days old.

\section{APPARATUS}

The arms of the wooden T-maze used were $2 \mathrm{ft}$ long, $4 \mathrm{in}$. wide and 5-10/16 in. high, and were covered with wire mesh. The start box, a continuation of the straight alley, was separated from the maze by a wooden door, which was hand operated vertically, as were the doors of the goal boxes. The right arm and goal box of the maze were painted glossy black. The left arm and goal box were painted flat white. The straight alley floor was painted glossy black on the right half and flat white on the left half. The left goal box contained a dry-food reward, which was placed on the floor. The right goal box contained the water reward, which was supplied by a standard water bottle.

The dry food used in the maze and home cages was standard Purina rat pellets. The liquid diet was vanilla Metrecal, which earlier testing in this laboratory had indicated that rats preferred to Purina pellets.

\section{PROCEDURE FOR GROUP S}

On each of the two days prior to maze training each rat was allowed five runs of the maze while in a nondeprived state. Next, the rats were trained for 20 days to turn left in the maze to get dry food and turn right to get water. A 22-h deprivation schedule was used to establish both the hunger and thirst drives, with free access to the nondeprived substance. The training period consisted of 10 days of food deprivation and 10 days of water deprivation, with one day of each type of deprivation occurring in each of 10 randomly ordered pairs of days.

Five correct trials per day were obtained using a noncorrection procedure. When a correct response was made a rat was allowed to consume the reward for 15 sec. Hence, each rat made a total of 50 reinforced right responses and 50 reinforced left responses, insuring equal habit strengths for the two turning responses.

At the end of the training trials each rat was deprived of food and water for $60 \mathrm{~h}$, then given free test trials in the maze. Food and water were in the goal boxes. A rat was allowed to remain in the goal box $15 \mathrm{sec}$ after each choice.

PROCEDURE FOR GROUP L

The procedure for the rats raised on the liquid diet was identical to that of Group $S$ until the third day of maze training. Rats in Group L would not drink water in the goal box, apparently because thirst reduction was achieved from the available Metrecal during a 22-h water deprivation period. For the next eight days forced trials were used, with rats being deprived of both Metrecal and water for $22 \mathrm{~h}$ before the training trials. The rats still consumed the food but not the 
water. In addition, all rats were weakened, and seven died, apparently from unanticipated effects of living on a Metrecal diet. Consequently, the forced trials were discontinued. Metrecal was removed, and dry food and water were supplied for two days, during which time no training trials were given. Although two more rats died, the remaining 12 showed immediate recovery, and the remaining 10 days of training were given in the same manner as for Group S. In all other respects the treatment for Group $L$ was identical to that for Group S. The procedure for Group $L$ resulted in 50 reinforced left responses toward food and less than 50 reinforced right responses toward water. Testing of response choice was made in the same manner as for Group S.

\section{RESULTS}

Two of the 12 rats in Group $L$ were eliminated from the analysis of results prior to giving the test trials because it was questionable whether they had learned the appropriate responses. However, these two rats also received test trials.

The percentage of errors made on the first trial of each of the days of training was figured for each deprivation state for the two groups. During the last two days of training to turn right toward water while on a water deprivation schedule no errors were made by rats in either group. On the first trials of the last two food deprivation days Group $S$ made 5 per cent errors and Group L made 15 per cent errors. The percentages indicate that both groups learned to make both responses correctly, but that the water response was learned slightly better by both groups.

On the five test trials, administered after $60 \mathrm{~h}$ of food and water deprivation, each rat in Group $S$ made five consecutive food responses. Considering only the choice on the first test trial for the 20 rats of Group $S$, it is clear that significantly more food than water responses were made.

On the five test trials the 10 remaining rats of Group $L$ made 22 turns toward the water and 28 turns toward food. Considering only the first test trial, six rats turned toward food and four toward water. A binomial test shows that the probability of this outcome does not differ significantly from that expected by chance.

A Fisher exact probability test was performed to compare the first trial choices of the two groups. The probability of the observed results occurring by chance is less than .01 . Group $S$ made a significantly higher proportion of food responses than Group L.

Since the two rats of the $\mathrm{L}$ group that were excluded from the previous analysis did receive test trials, results with their data included were examined. Over all five test trials there were 31 food and 29 water responses. On the first test trial there were six food and six water responses. Inclusion of the excluded data makes the chance performance of Group L and the significant difference between the two groups even more striking.

\section{DISCUSSION}

Test trials were conducted after $60 \mathrm{~h}$ of simultaneous deprivation partly because of the extremely weak condition of rats deprived for more than $60 \mathrm{~h}$. In addition, Crawford \& James (1964) found $72 \mathrm{~h}$ to be the point of equality of hunger and thirst drives for rats 95 days old. Rats in the present experiment were approximately 65 days old at the time of testing. For rats younger than 95 days the expectation is that the point of equality of drives would occur earlier than $72 \mathrm{~h}$, assuming that the relation of the gradients for hunger and thirst found by Crawford and James is similar for younger rats. In the present experiment it is probable that the point of equality of drives had been passed by $60 \mathrm{~h}$, considering the weakened condition of the animals. Thus, according to the findings of Crawford and James, the thirst drive was probably slightly stronger for both groups of rats.

The $S$ group gave 100 per cent food responses on the test trials. This result is consistent with previous findings of Heron (1949) and Manning (1954). A plausible explanation of these results is given by the Hull-Spence theory. The 100 per cent food responses occur because of a stronger incentive value for eating than drinking established during the normally raised rats' lives.

The prediction that Group $\mathrm{S}$ would give a significantly higher percentage of food responses than Group $L$ was confirmed. Although some procedural changes for Group L were necessarily enacted during the training period, none of the changes affected the prediction that the two groups would differ on the test trials. Raising rats for most of their lives on a liquid diet increased the amount of drinking time, lengthened the period of reinforcement associated with drinking, and, according to the Hull-Spence theory, should have developed a greater incentive value for the drinking response. The significantly greater percentage of turns toward water by Group L supports the prediction of the theory.

The prediction that Group L would make significantly more water than food responses was not confirmed. Perhaps if rats in Group $L$ had remained on the liquid diet they would have made more water responses. The use of dry food in the home cage for the last 12 days of the experiment should have increased the incentive value of the eating response, causing Group $\mathrm{L}$ to make fewer water responses than would otherwise have occurred.

The change from free trials to forced trials and back again to free trials during the training period should not have affected the results of the test trials, as long as the training procedure resulted in the rats' learning to make different responses on the basis of drive discrimination. As previously indicated, rats of Group L did learn the discrimination.

The loss of nine rats from Group $L$ poses no problem for interpreting the choice data, since it is improbable that there was any systematic relationship between survival and choice in the T-maze.

It would be interesting to determine the choice behavior of rats raised on a liquid diet throughout training and test trials. Perhaps, as the Hull-Spence theory would predict, the percentage of turns toward water would increase above the chance level observed in this experiment.

\section{REFERENCES}

CRAWFORD, M. L. J., \& JAMES, W. T. Effect of acute simultaneous food and water deprivation upon bar pressing behavior. Psychological Reports, 1964, 15, 239-242.

HERON, W. T. Internal stimuli and learning. Journal of Comparative \& Physiological Psychology, 1949, 42, 486-492.

HULL, C. L. Differential habituation to internal stimuli in the albino rat. Journal of Comparative \& Physiological Psychology, 1933, 16, 255-273.

KLEINMUNTZ, B. One response learned to two drives. Journal of General Psychology, 1963, 68, 181-186.

LEEPER, $R$. The role of motivation in learning. Journal of Genetic Psychology, 1935, 46, 3-40.

MANNING, $H$. M. The effects of varying conditions of hunger and thirst on two responses learned to hunger or thirst alone. Unpublished doctoral dissertation, University of Minnesota, 1954.

SPENCE, K. W. Behavior theory and conditioning. New Haven: Yale University Press, 1956.

\section{NOTES}

1. This article is based on an experiment conducted as an M.A. thesis by the first author under supervision of the second author.

2. Presently at the University of Mississippi. 